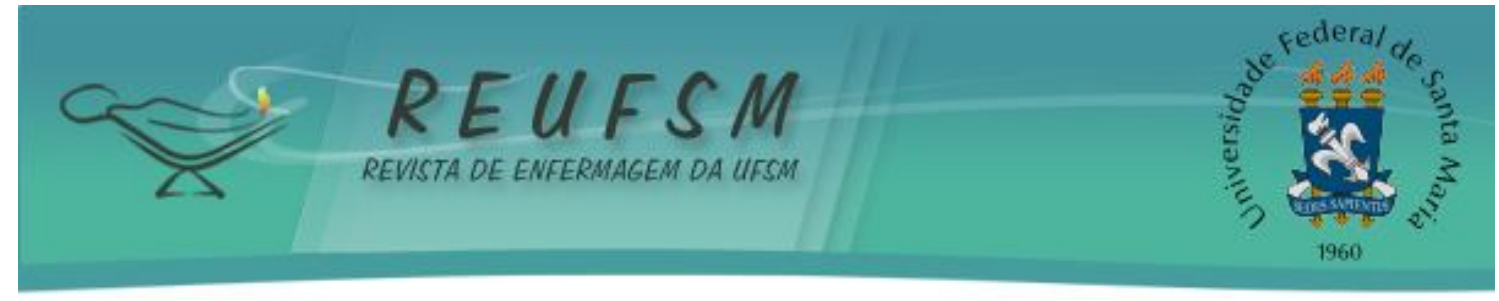

ARTIGO DE REVISÃO

\title{
A PRODUÇÃO CIENTÍFICA BRASILEIRA SOBRE CUIDADOS DE ENFERMAGEM A PACIENTES PORTADORES DE ÚLCERAS POR PRESSÃO
}

\section{THE BRAZILIAN SCIENTIFIC PRODUCTION OF NURSING CARE TO PATIENTS WITH PRESSURE ULCERS \\ LA PRODUCCIÓN CIE NT ÍFICA BRASILEÑA EN LA ATENCIÓN DE ENFERMERÍA A PACIE NTES CON ÚLCERAS POR PRESIÓN}

\author{
Rodrigo Marques da Silva ${ }^{1}$ \\ Roger Rodrigues Peres ${ }^{2}$ \\ Crhis Netto de Brum ${ }^{3}$ \\ Silviamar Camponogara ${ }^{4}$
}

RESUMO: Objetivo: conhecer o que tem sido produzido sobre Úlceras por Pressão, visando a tecer reflexões sobre esse tema no contexto da assistência à saúde. Método: realizou-se uma revisão bibliográfica, de abordagem quantitativa, no SciELO, BDENF e LILACS, por meio do descritor Úlcera por pressão e no idioma português. Incluíram-se as produções em língua portuguesa publicadas no período de 1999 a 2010. Elaborou-se um quadro sinóptico, avaliando-se a categoria profissional do primeiro autor de cada publicação, metodologia, fonte, sujeitos pesquisados e área de conhecimento dos sujeitos estudados e temática principal do estudo. Resultados: das produções analisadas, 47,14\% utilizam pesquisas de campo como metodologia. Prevaleceram produções com pacientes, sendo $68,57 \%$ destes internados em hospitais, $14,28 \%$ do total, envolvendo profissionais de saúde, e $2,85 \%$ com estudantes de enfermagem. Conclusão: a literatura brasileira tem produzido poucas pesquisas voltadas para a discussão do ensino-aprendizagem no tratamento e prevenção das UPP.

Descritores: Cuidados de enfermagem; Estudantes de enfermagem; Úlcera por pressão.

ABST RACT: Objective: to know what has been produced on pressure ulcers in order to make reflections on this theme in health care Method: it was a bibliographic review, a quantitative approach, in SciELO, BDENF and LILACS, through the descriptor Pressure ulcer and the Portuguese language. Productions in Portuguese published from 1999 to 2010 were included. A summary table was drawn up, to assess the professional category of the first author of each publication, methodology, source, research subjects, areas of knowledge of subjects study and main theme of the study. Results: From the productions found, $47.14 \%$ use field research. Productions with patients stood out, $68.57 \%$ of those admitted to hospitals, $14.28 \%$ of total involving health professionals and $2.85 \%$ nursing students. Conclusion: The Brazilian literature has produced little research for the discussion of teaching and learning in the treatment and prevention of pressure ulcers.

Descriptors: Nursing care; Students nursing; Pressure ulcer.

\footnotetext{
${ }^{1}$ Acadêmico do 70 Semestre do Curso de Enfermagem da Universidade Federal de Santa Maria. E-mail: marques-sm@hotmail.com

${ }^{2}$ Acadêmico do $7^{0}$ Semestre do Curso de Enfermagem da Universidade Federal de Santa Maria. E-mail: roger_rrp@yahoo.com.br

3 Enfermeira. Especialista em Saúde Coletiva. Mestranda do Programa de Pós-Graduação em Enfermagem da Universidade Federal de Santa Maria. Bolsista CAPES. E-mail: crhisdebrum@gmail.com

${ }^{4}$ Enfermeira. Doutora em Enfermagem. Professora Adjunta do Departamento de Enfermagem da Universidade Federal de Santa Maria Membro do Grupo de Pesquisa Trabalho, Educação, Saúde e Enfermagem. E-mail: silviaufsm@yahoo.com.br
} 


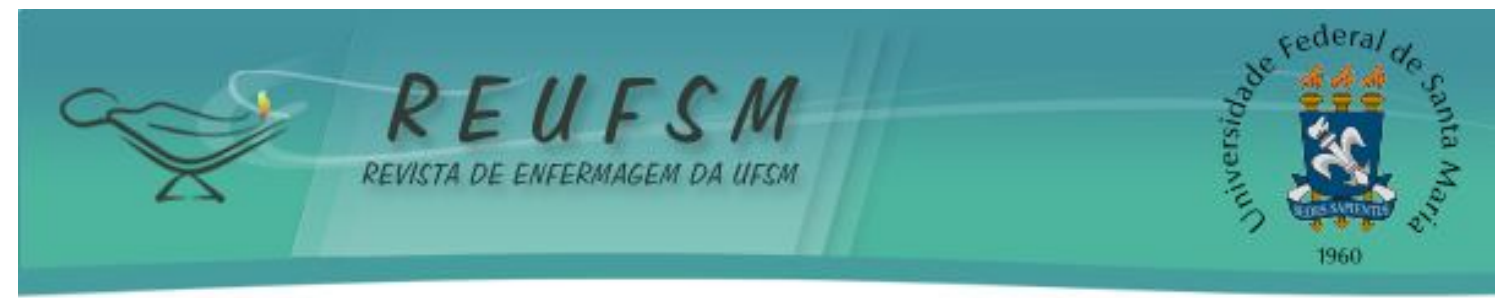

RESUMEN: Objetivo: Conocer lo que se ha producido acerca de las úlceras por presión, con el fin de reflexionar sobre el tema en el entorno de la asistencia a la salud. Método: una revisión bibliográfica, con enfoque cuantitativo, en las bases SciELO, LILACS y BDENF, por medio del descriptor Úlcera por pressão. Se incluyeron las producciones en lengua portuguesa publicadas en el periodo 1999-2010. Se hizo una tabla de resumen en la que se consideraron la evaluación de la categoría profesional del primer autor de cada publicación, metodología, fuentes, temas de investigación, áreas de conocimiento de los sujetos estudiados y tema principal del estudio. Resultados: De las producciones analizadas, el 47,14\% utilizó la investigación de campo como metodología. Predominaron producciones con los pacientes, el $68,57 \%$ internados en un hospital, el $14,28 \%$ con participación de profesionales de la salud y el 2,85\% con estudiantes. Conclusión: Poco se ha producido para la discusión de la enseñanza y el aprendizaje en el tratamiento y prevención de la UPP.

Descriptores: Atención de Enfermería; Estudiantes de enfermería; Úlcera por presión.

\section{INTRODUÇÃO}

Atualmente, o paciente internado em hospitais de grande ou pequeno porte, e, até mesmo, assistido em domicílio, pode desenvolver lesões cutâneas de diversas etiologias. Nesse contexto, ressalta-se a relevância de um estudo, realizado junto a prontuários de 1.120 pacientes internados em um hospital filantrópico, o qual evidenciou que grande parte $(42,3 \%)$ dos pacientes estudados desenvolveu lesões cutâneas, durante 0 período de hospitalização, de forma que as feridas operatórias $(88,6 \%)$ e as úlceras por Pressão $(9,9 \%)$ tiveram as maiores taxas de ocorrência. ${ }^{1}$

0 paciente internado requer uma atenção especial, quando se trata de lesões de pele, por este apresentar, de forma agregada ou isolada, ferimentos de etiologia cirúrgica (incisão ou excisão); traumática (agressão mecânica, térmica ou química) e crônica (fisiopatologias subjacentes, por exemplo, a Úlcera por Pressão). ${ }^{2}$

Dentre as principais lesões cutâneas, estão as Úlceras por Pressão (UPP), que são áreas de morte celular localizadas na pele e tecidos subjacentes, causadas por pressão, cisalhamento, fricção e (ou) a combinação destes. ${ }^{3}$ Entretanto, é importante considerar que existe divergência entre autores sobre a conceitualização dos estágios da UPP, pois, para alguns, o estágio 1 ocorre, quando, ainda, não há o rompimento da integridade da pele, mas a presença de hiperemia no local de pressão, fato que, para outros autores, não indica presença de ulceração. ${ }^{3} 0$ Ministério da Saúde, por sua vez, considera a lesão em estágio 1, quando há presença de hiperemia não reativa, sem rompimento da integridade cutânea; estágio 2, quando ocorre rompimento da epiderme e derme; estágio3, quando ocorre rompimento do tecido subcutâneo, podendo estender-se até a fáscia muscular; estágio 4, quando acomete, desde a fáscia muscular até músculos e ossos. ${ }^{2}$

A presença de Úlcera por Pressão tem sido considerada um indicador de qualidade da assistência de enfermagem nos serviços de saúde, subjazendo esforços para estabelecer-se diretrizes e protocolos que norteiam a prática, buscando a redução desse problema, tanto nos hospitais brasileiros quanto no restante do mundo. ${ }^{4}$

Dessa forma, dentre os vários profissionais, que compõem a equipe de saúde, tem sido incumbida, ao enfermeiro, a responsabilidade pela avaliação das UPPs, lembrando que, para a realização de um cuidado integral, exige-se a observação de alguns fatores envolvidos no processo de cicatrização, incluindo: a nutrição, o estado emocional, a idade, a dieta, a fragilidade vascular e a alteração da mobilidade, não se restringindo aos produtos utilizados como coberturas. 


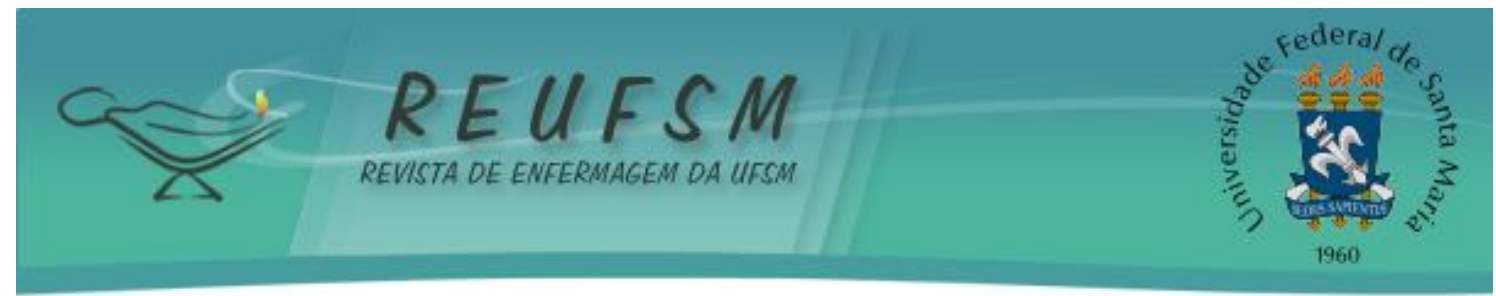

Assim, a abordagem desses aspectos remete-nos a reflexões sobre a formação profissional dos estudantes de enfermagem, que buscam a qualificação necessária, durante os cursos de graduação ofertados pelas diversas instituições de ensino superior existentes no Brasil. Particularmente, há uma preocupação com a abordagem da temática em questão, durante o processo formativo, tendo em vista que esses conhecimentos são parte do cotidiano do enfermeiro, em diversos cenários de atuação, os quais precisam ser contemplados, tanto do ponto de vista teórico, quanto do prático. Como o enfermeiro é um profissional que deve possuir um saber teórico e prático, o seu processo de formação acadêmica necessita contemplar, obrigatoriamente, o ensino teórico e as habilidades práticas necessárias à sua formação, o que ocorrerá nos campos de aulas práticas. ${ }^{5}$

Sabidamente, a produção de conhecimentos de determinada área de atuação pode ser considerada um indicador dos rumos que norteiam a formação profissional. Dessa forma, apresenta-se este estudo, que traz como inquietação principal a produção científica brasileira sobre Úlceras por Pressão, no campo da saúde. Dessa forma, constituise em objetivo: conhecer o que tem sido produzido sobre Úlceras por Pressão, visando a tecer reflexões sobre esse tema no contexto da assistência à saúde.

\section{MÉTODO}

Realizou-se uma pesquisa bibliográfica, entendida como o ato de indagar e de buscar informações sobre determinado assunto, através de um levantamento realizado em base de dados nacionais e estrangeiras, com o objetivo de detectar o que existe de consenso ou de polêmico no estado da arte da literatura. ${ }^{6}$

$O$ estudo tem abordagem quantitativa, descritiva e foi realizado nas seguintes bases de dados: Base de Dados de Enfermagem (BDENF), Literatura Latino-Americana e do Caribe em Ciências da Saúde (LILACS), Scientific Electronic Library On-line (SciELO), destacando-se que esta última, no período da coleta, apresentava apenas produções indexadas até 0 ano de 2008.

A busca foi desenvolvida utilizando-se o descritor de assunto Úlcera por Pressão, indexado ao DECS (Descritores de Ciências da Saúde), a qual foi realizada no formulário avançado das bases de dados, anteriormente citadas, utilizando-se a conjunção and entre o campo descritor de assunto e o idioma nesses formulários.

Os dados foram coletados no período de maio a junho de 2010. Utilizou-se o recorte temporal, entre os anos de 1999 a 2010, tendo como critério de inclusão as produções com resumos em língua portuguesa publicados nesse período. Realizou-se esse recorte temporal, a fim de conhecer as produções mais atuais, ressalvando-se que as publicações do ano de 2010 estão incompletas, visto que, ainda, estão em andamento. Os critérios de exclusão foram: os artigos de língua inglesa e espanhola, que envolveram animais e os que não trabalharam diretamente com Úlceras por Pressão.

$\mathrm{Na}$ coleta de dados, buscaram-se publicações que tivessem como tema principal as Úlceras por Pressão e elaborou-se um quadro sinóptico, a fim de avaliar os seguintes aspectos em relação às produções: categoria profissional do primeiro autor de cada publicação, buscada no Sistema de Currículos Lattes, metodologia, fonte, sujeitos pesquisados, área de conhecimento dos sujeitos estudados, quando estes fossem profissionais de saúde e temática principal do estudo.

Posteriormente à coleta de dados, os resumos das publicações foram analisados e quantificados, por meio de estatística simples, conforme os aspectos do quadro sinóptico supracitado. Os resultados numéricos foram agrupados e apresentados em escala de frequência e percentual, sendo possível fazer uma análise das temáticas envolvidas nas produções. Os resultados do estudo estão apresentados no item a seguir. 


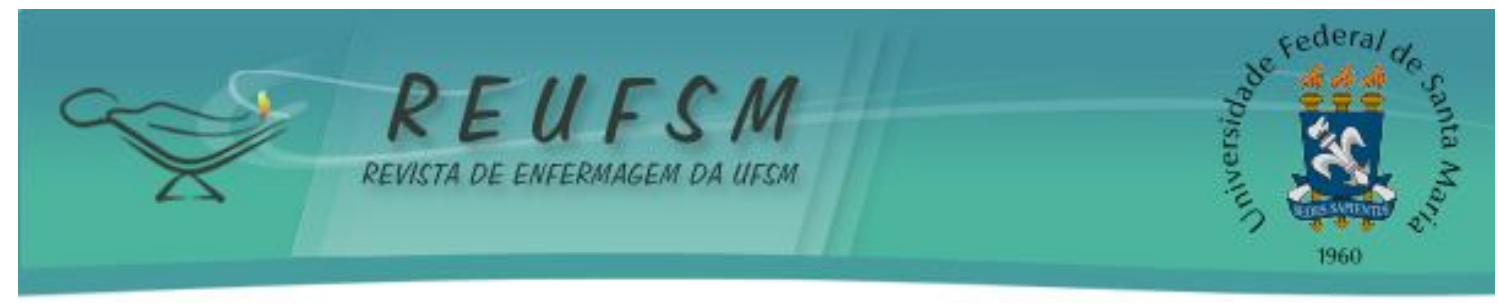

\section{RESULTADOS}

Dos 100 resumos encontrados na base de dados LILACS, com o descritor mencionado, foram excluídos da pesquisa 63 resumos. Sendo 30 destes excluídos, por terem sido produzidos anteriormente ao ano de 1999; um resumo, por já estar presente na SciELO; nove deles, por não tratarem diretamente sobre UPP e o restante (23 resumos), por já estarem presentes na Base de Dados da Enfermagem (BDENF), sendo assim, foram utilizados 37 resumos da base de dados em questão. No SciELO, foram encontrados seis resumos, porém, foram incluídos na pesquisa somente dois, pois os outros (quatro) não tratavam, explicitamente, de Úlceras por Pressão. Na BDENF, foram encontrados 63 resumos, dos quais foram utilizados apenas 31, sendo excluídos dez, por já estarem presentes na própria base de dados; cinco, por não tratarem diretamente da temática pesquisada e 17, por estarem fora do recorte temporal delimitado pelo estudo. Dessa forma, foi incluído nesta pesquisa um total de 70 resumos, que, após a contagem, foram analisados e quantificados.

Quanto à metodologia dos resumos, observou-se que $47,14 \%$ das produções têm utilizado pesquisas de campo como metodologia de estudo. As revisões bibliográficas, também, apresentaram uma frequência notável, correspondendo a $12,85 \%$ dos resumos encontrados. Abaixo destas seguem os relatos/estudos de caso, com $8,57 \%$ relatos de experiência, com $5,71 \%$ e os resumos, com outras metodologias, corresponderam a $7,14 \%$ dos achados. Além disso, os artigos de atualização, estudos de validação e revisões integrativas foram as produções menos encontradas, com $1,42 \%$ do total de resumos. Vale destacar que $14,28 \%$ dos resumos não disponibilizavam a metodologia utilizada, o que dificultou a realização de uma contagem efetiva para as categorias descritas.

Quanto às fontes das produções científicas, pode-se dizer que as dissertações de mestrado apresentaram a maior frequência de publicação com a temática pesquisada, sendo esta de $21,42 \%$ o equivalente a 15 resumos. A Revista Latino-Americana de Enfermagem concentrou 7,14\% (cinco resumos) das publicações. As demais revistas tiveram entre um e quatro resumos indexados às bases analisadas.

Quanto à categoria profissional dos autores, considerando-se, apenas, o primeiro autor de cada produção, observou-se que $77,78 \%$ eram enfermeiros, seguidos por médicos $(12,96 \%)$. Os profissionais formados em Odontologia, Letras e Fisioterapia, e estudantes de enfermagem corresponderam a $1,86 \%$ do total dos autores. Além disso, o currículo de um dos autores não foi encontrado no Sistema de Currículo Lattes.

A partir dos objetivos, constatou-se que há um predomínio dos estudos envolvendo as temáticas sobre o uso de técnicas e cuidados, voltados à prevenção e cura das úlceras por Pressão, com o processo de instrumentalização dos trabalhadores e com conhecimento da equipe frente ao tratamento destas. Alguns dos cuidados mais relatados nos resumos foram: uso de almofadas flexíveis; aplicação das escalas de Braden, de Waterlow, de Norton e de Gosnell; uso tópico de Triglicerídeos de Cadeia Média (TCM), de clara de ovo, de solução glicerinada, de AGE (Àcidos Graxos Essenciais), de óleo de girassol; alternância de decúbito e avaliação do estado nutricional.

Outras técnicas observadas nos resumos selecionados pela pesquisa foram procedimentos que visam à cura da úlcera já estabelecida, como: retalhos cutâneos e musculoesqueléticos (da coxa, transverso-lombar, fáscio-cutâneo em V-Y); utilização de medicamentos e materiais (placas hidrocoloides, uso da (das) pomada (pomadas) Iruxol, Hidrogel e Alginato de Cálcio) e também o uso do laser AsALGa, 830nm.

Quanto aos sujeitos de pesquisa, observou-se que, em 35 resumos abordaram-se pacientes, sendo $68,57 \%$ destes internados em hospitais. Os pacientes com internação, de 


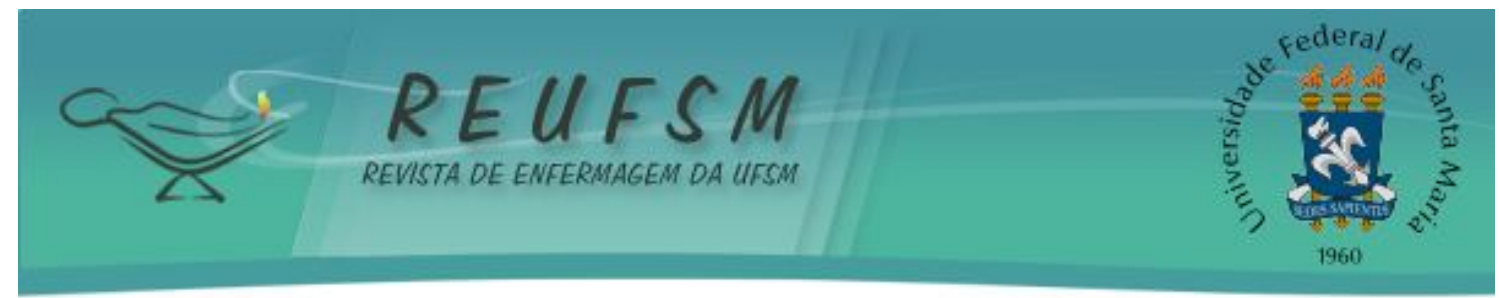

Ionga permanência, em casas de repouso, tiveram a segunda maior frequência $(8,57 \%)$, seguidos pelos estudos com pacientes internados em domicílio $(5,71 \%)$.

Os resumos que trabalharam com pacientes internados em Centros de Reabilitação e aqueles que trabal haram com outros suj eitos, que não estavam presentes nas categorias elaboradas, corresponderam a $2,85 \%$

As produções, que trabalharam com profissionais de saúde, corresponderam a $14,28 \%$ sendo $90,0 \%$ deles enfermeiros, ou outro integrante da equipe de enfermagem, e $10,0 \%$ tinham médicos como sujeitos de pesquisa. Os estudantes de enfermagem foram sujeitos de estudo em dois artigos (2,85\%), presentes em uma das bases de dados pesquisadas, sendo que um resumo enfocava os cuidados preventivos de enfermagem e, o outro, cuidados preventivos e terapêuticos ao portador de UPP, ambos com a visão voltada para a área hospitalar.

\section{DISCUSSÃO}

Os dados revelam que a maior parte das produções tem utilizado pesquisas de campo e revisões bibliográficas como metodologia. Dentre as revisões, observou-se um número reduzido de pesquisas que utilizaram a revisão integrativa da literatura como método, a qual é de suma importância para a enfermagem, já que permite a busca, a avaliação crítica e a síntese das evidências disponíveis do tema investigado, sendo o produto final o estado atual do conhecimento, a implementação de intervenções ef etivas na assistência à saúde e a redução de custos. ${ }^{7}$

Embora parte dos estudos esteja relacionada a pesquisas de campo, observa-se que poucas correspondem à estratégia do estudo de caso, a qual é fundamental para o exercício de inter-relação entre teoria e prática, qualificando o processo formativo. Entende-se que, 0 uso dessas estratégias de ensino, acrescidas do estímulo a sua divulgação, são essenciais para a formação do futuro profissional de enfermagem. Dessa forma, a inter-relação teoria e prática é uma das estratégias de ensino-aprendizagem mais apropriada para a formação profissional, pois oportuniza que o acadêmico estabeleça relações entre as experiências que vivencia e os conteúdos teóricos que Ihe dão suporte.

Em $50 \%$ dos estudos, os suj eitos foram pacientes, sendo $68,57 \%$ destes internados em hospitais. Pode-se afirmar que o foco das pesquisas ainda tem sido o nível terciário de atenção à saúde, ou seja, hospitais, em geral, de grande e médio porte, sendo observados poucos estudos $(5,71 \%)$ com pacientes em domicílio e nenhum envolvendo Unidades Básicas de Saúde (UBS) e (ou) Estratégias de Saúde da Família (ESF). Isso demonstra que há poucos estudos abordando o cuidado de enfermagem a pacientes portadores de úlceras por Pressão em domicílios e acerca daqueles que buscam cuidados de enfermagem nas UBS e ESF. Quando nos remetemos as UBS e ESF, estamos falando em englobar, não somente, a cura das doenças, mas a sua prevenção e o direcionamento de ações que venham a resgatar o completo bem-estar físico, mental, social e espiritual. ${ }^{8} \mathrm{~A}$ partir disto, pode-se enfatizar que realizar estudos sobre a ocorrência e intervenções de enfermagem aos portadores de Úlcera por Pressão, neste nível de atenção à saúde, é extremamente relevante, embora não seja uma realidade das produções científicas sobre o tema, como constatado no estudo. É importante compreender que uma atenção de qualidade e efetiva, ao público em questão, neste nível de atendimento, pode proporcionar um menor agravamento das feridas, minimizando a possibilidade de uma intervenção em nível terciário, e, por consequência, os prejuízos físicos e psicológicos destes pacientes.

Os profissionais da saúde, enquanto sujeitos da pesquisa, compreendem dez das publicações, sendo que nove são trabalhadores da área da enfermagem, demonstrando, assim, uma maior preocupação quanto aos saberes destes trabalhadores referente às UPP, 


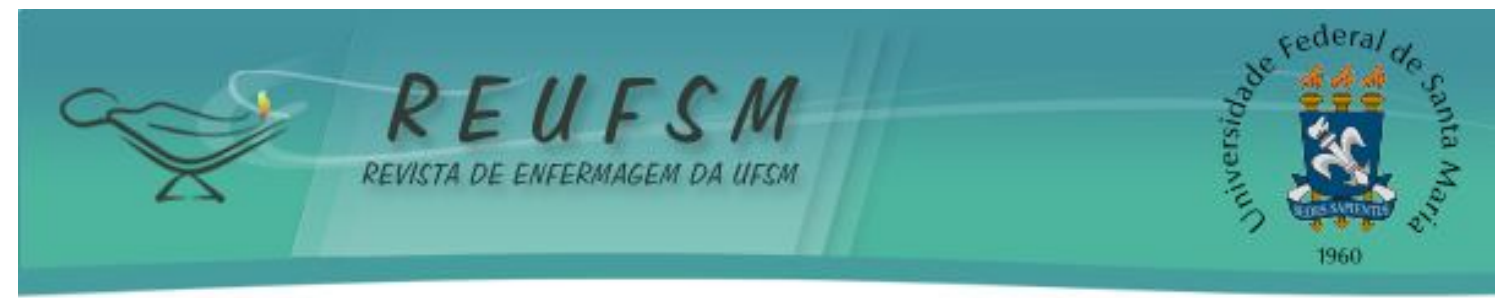

uma vez que nessa área (das alterações cutâneas) a ciência vem renovando-se com muita frequência, exigindo do profissional a busca constante do conhecimento, além de se tratar de um tema que exige atuação ativa do enfermeiro, sendo, inclusive, objeto de cursos de especialização. ${ }^{9}$

Constatou-se que há um predomínio dos estudos envolvendo as temáticas sobre o uso de técnicas e cuidados, voltados à prevenção e à cura das Úlceras por Pressão, com o processo de instrumentalização dos trabalhadores e o com o conhecimento da equipe frente ao tratamento destas. ${ }^{10}$

Em nosso país, o movimento da prática baseada em evidências na enfermagem é incipiente e a maioria da literatura disponível não é nacional. Porém, ao mesmo tempo, percebe-se que é uma área em potencial desenvolvimento, o que amplia a possibilidade de expansão de nossa prática profissional. ${ }^{11}$

Com relação aos graduandos de enfermagem, pode-se destacar que existem reduzidos estudos que têm como finalidade conhecer o ponto de vista dos acadêmicos de enfermagem, frente à prevenção e ao tratamento das UPP e/ ou analisar as propostas curriculares vigentes nos cursos de graduação das instituições de ensino superior, visando a uma melhor qualificação destes acadêmicos. Isto vem ao encontro do exposto por alguns autores, que apontam para a necessidade de uma revisão do ensino e do perfil profissional, buscando adequar a formação do enfermeiro à realidade social de cada região e a organização do setor saúde, tendo em vista as mudanças ocorridas na enfermagem, nas últimas décadas, na América Latina e, sobretudo, no Brasil. ${ }^{12}$

Dentre as fontes de publicação, o estudo revelou que a maior quantidade dos achados encontra-se no meio acadêmico, através de dissertações de mestrado, indicando que a maior parte das publicações têm sido produzidas por pós-graduandos. Tais resultados são reforçados por alguns autores, ao enfatizarem que o campo de desenvolvimento da pesquisa e 0 avanço do conhecimento científico em enfermagem vêm ocorrendo, de modo prioritário, em ambientes acadêmicos, tendo sua história construída com algum trânsito no campo da prática. ${ }^{13-14}$

Diante desse contexto, verificou-se que a categoria profissional que mais se preocupa com essa temática, em suas pesquisas, são os enfermeiros, especialmente os pósgraduandos. Este achado pode estar diretamente relacionado à atribuição da culpa e responsabilidade da ocorrência dessas lesões ao enfermeiro, justificando que a presença ininterrupta da enfermagem no cenário hospitalar remete-a a maior responsabilidade sobre 0 cuidado e com os resultados do processo assistencial. ${ }^{4}$ Entretanto, com 0 desenvol vimento dos estudos e da tecnologia, as evidências científicas têm mostrado que a Úlcera por Pressão não é de responsabilidade apenas da enfermagem, devido aos fatores múltiplos ou a multicausalidade da sua ocorrência. ${ }^{15}$ Desta maneira, acredita-se que este cuidado é inerente a todos os profissionais que participam do processo de cuidar do paciente. No entanto, esta preocupação possibilita a produção de novos conhecimentos para a profissão, bem como a promoção da melhoria do cuidado ao paciente, visto os desafios constantemente enfrentados pelos enfermeiros. ${ }^{7}$

\section{CONCLUSÃO}

Esse estudo possibilitou concluir que a literatura brasileira tem produzido um reduzido número de pesquisas voltadas à discussão do ensino-aprendizagem no tratamento e na prevenção das UPP, com os acadêmicos de enfermagem, público este que requer uma atenção diferenciada, pois serão os futuros profissionais inseridos em um mercado de trabalho cada vez mais exigente e competitivo. É importante que os estudantes de enfermagem estejam envolvidos em um processo de ensino-aprendizagem, que abarque 


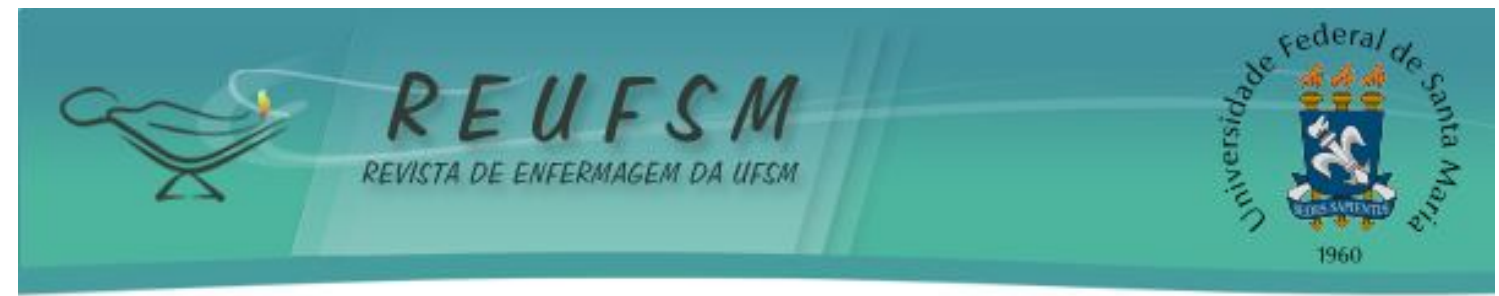

diversos aspectos de sua formação, assim como, sejam estimulados a participar de atividades de pesquisa como autores e futuros enfermeiros, em busca de conhecimento por meios adequados de obtenção de informações.

A maior parte das produções envolve pacientes em âmbito hospitalar com foco curativo, necessitando-se de mais estudos junto a usuários dos serviços de atenção primária à saúde, sob um enfoque preventivo e (ou) terapêutico. Além disso, sugere-se maior número de estudos de campo, que auxiliem na melhoria da qualidade da assistência prestada e na redução de custos, além de fortalecer a enfermagem enquanto ciência pautada em evidências.

Considera-se, como principal limitação do estudo, o fato de muitos resumos não estarem adequadamente formulados, deixando dúvidas sobre o método utilizado, bem como sobre os resultados.

A recomendação é de que haja a produção de maior número de estudos relacionados a essa temática, os quais, além de fortalecerem a profissão, enquanto campo do conhecimento, poderão melhor subsidiar o processo formativo, já que o enfermeiro é o profissional que tem maior participação no processo de cuidado a pessoas portadoras de Úlceras por Pressão. Da mesma forma, o enfermeiro também tem grande responsabilidade no desenvolvimento de ações preventivas de tais lesões, tanto em nível hospitalar quanto comunitário.

\section{REFERÊNCIAS}

1. Maciel, EAF. Prevalência de feridas em pacientes internados em um hospital filantrópico de grande porte de Belo Horizonte [dissertação]. Minas Gerais (MG): Escola de Enfermagem da Universidade Federal de Minas Gerais. Universidade Federal de Minas Gerais; 2008.

2. Ministério da Saúde. Secretaria de Assistência à Saúde. Instituto Nacional de TraumatoOrtopedia. Caderno de Enfermagem em Ortopedia. Curativos- Orientações Básicas. Brasília; 2006 [citado em: 3 jul. 2010]. Disponível em: http:// bvsms. saude.gov. br/bvs/ publicacoes/caderno_enfermagem_ortopedia. pdf.

3. Prazeres SJ. Silva, ACB. Tratamento de feridas: teoria e prática. In: Prazeres SJ, organizadora. Úlceras por Pressão. $1^{a}$ ed. Porto Alegre: Moriá; 2009. p.112-38.

4. Carvalho LS, Ferreira SC, Silva CA, Santos ACPO, Regebe CMC. Concepções dos acadêmicos de enfermagem sobre prevenção e tratamento de úlceras por pressão. Rev Baiana Saúde Pública. 2007 jan-jun; 31(1): 77-89.

5. Salomé GM, Espósito VHC. Vivências de acadêmicos de enfermagem durante o cuidado prestado às pessoas com feridas. Rev Bras Enferm. 2008 nov-dez; 61(6): 822-7.

6. Bastos OM, Deslandes SF. Sexualidade e o adolescente com deficiência mental: uma revisão bibliográfica. Ciência e Saúde Coletiva. 2005 abr-jun; 10(2): 389-97.

7. Mendes KDS, Silveira RCCP, Galvão CM. Revisão integrativa: método de pesquisa para a incorporação de evidências na saúde e na enfermagem. Texto e Contexto Enferm. 2008 out-dez; 17(4): 758-64.

8. Martins JJ, Nascimento ERP, Erdmann AL, Candemil MC, Belaver GM. O cuidado no contexto domiciliar: o discurso de idosos/familiares e profissionais. Rev Enferm UERJ. 2009 out/ dez; 17(4): 556-62.

9. Marin MJ S, Vilela EM, Takeda E, Santos IF. Rev Esc Enferm USP. 2002; 36(4): 338-44. 


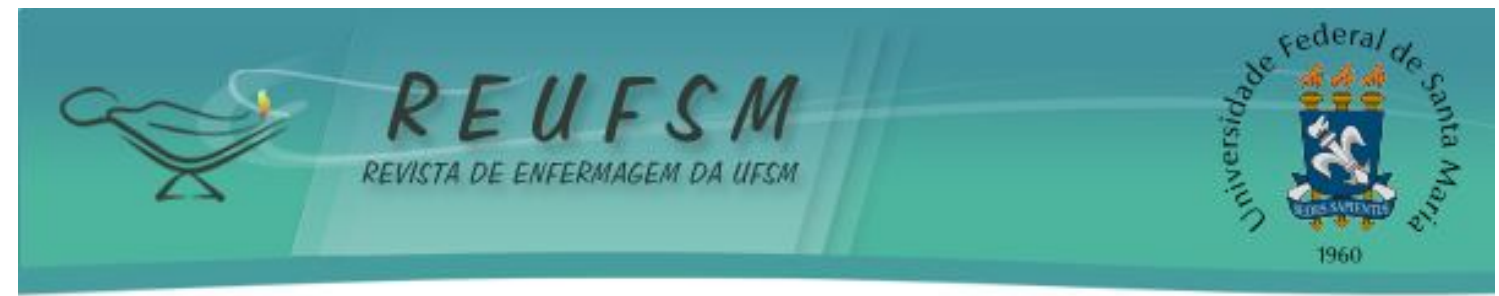

10. Moura GMSS, Juchem BC, Falk MLR, Magalhães AMM, Suzuki LM. Construção e implantação de dois indicadores de qualidade assistencial de enfermagem. Rev Gaúcha Enferm. 2009 mar; 30(1):136-40.

11. Aguiar DF, Camacho KG. O cotidiano do enfermeiro em pesquisa clínica: um relato de experiência. Rev Esc Enferm USP. 2010 jun; 44(2): 526-30.

12. Guariente MHDM. A experiência do NUPE- Núcleo de Pesquisa em Enfermagem do Hospital Universitário - UEL. Anais do 11ํㅡㄹ Congresso Brasileiro de Medicina Intensiva; 2004 jun. 13-15; Curitiba (PR); 2004.

13. Leão ER. Experiências em pesquisa com enfermeiras no Hospital Samaritano. Anais do 11ํㅡ Congresso Brasileiro de Medicina Intensiva; 2004 jun. 13-15; Curitiba (PR); 2004.

14. Silva MB da, Meneghete MC, Fontana RT. Implementação do processo de enfermagem na prática clínica: experiência de aprendizado. Rev Enferm UFPE [periódico da internet]. 2010 abr/jun[citado em: 3 jul. 2010]; 4(2):539-47. Disponível em: http:/ / www. ufpe. br/ revistaenfermagem/ index. php/revista/ article/ view/ 712/ pdf_43

15. Rabeh, SAN. Úlcera de pressão: a clarificação do conceito e estratégias para divulgação do conhecimento na literatura de enfermagem [dissertação]. Ribeirão Preto (SP): Escola de Enfermagem de Ribeirão Preto. Universidade de São Paulo; 2001.

Data de recebimento: 20/01/2011

Data de aceite: 06/04/2011

Contato com autor responsável: Rodrigo Marques da Silva. Rua Conde de Porto Alegre, 1240, apto 203. CEP 97015110. Santa Maria- RS.

E-mail: marques-sm@hotmail.com 